\title{
Quantum phase transition with a simple variational ansatz
}

\author{
Y. Lutsyshyn, ${ }^{1, *}$ G. E. Astrakharchik,${ }^{2, \dagger}$ C. Cazorla, ${ }^{3}$ and J. Boronat ${ }^{2, \dagger}$ \\ ${ }^{1}$ Institut für Physik, Universität Rostock, 18051 Rostock, Germany \\ ${ }_{2}^{2}$ Departament de Física i Enginyeria Nuclear, Universitat Politècnica de Catalunya, Campus Nord B4-B5, E-08034 Barcelona, Spain \\ ${ }^{3}$ School of Materials Science and Engineering, University of New South Wales, Sydney, NSW 2052, Australia \\ (Received 1 October 2014; revised manuscript received 26 November 2014; published 11 December 2014)
}

\begin{abstract}
We study the zero-temperature quantum phase transition between liquid and hcp solid ${ }^{4} \mathrm{He}$. We use the variational method with a simple yet exchange-symmetric and fully explicit wave function. It is found that the optimized wave function undergoes spontaneous symmetry breaking and describes the quantum solidification of helium at $22 \mathrm{~atm}$. The explicit form of the wave function allows us to consider various contributions to the phase transition. We find that the employed wave function is an excellent candidate for describing both a first-order quantum phase transition and the ground state of a Bose solid.
\end{abstract}

DOI: 10.1103/PhysRevB.90.214512

PACS number(s): 67.80.-s

Properties of solid ${ }^{4} \mathrm{He}$ have regained attention due to a host of unexpected physics discovered in the past decade [1-8]. Most of the new features occur close to absolute zero and are believed to be primarily driven by quantum effects. Consequently, the solidification of ${ }^{4} \mathrm{He}$ came under renewed scrutiny. The role of quantum statistics in the transition location has been recently revisited in Ref. [9]. At small but nonzero temperatures, indistinguishability of particles destabilizes the quantum solid. Distinguishable particles, on the other hand, would solidify even at low pressures, with the phase diagram reminiscent of the Pomeranchuk effect $[10,11]$. The feature was dubbed in [9] as thermocrystallization. A similar effect was seen numerically for the Wigner-crystallization of a two-dimensional Coulomb system [12]. The solidification of ${ }^{4} \mathrm{He}$ at zero temperature was revisited in Ref. [13] with the density functional theory (DFT). Results were improved compared with previous DFT studies.

In this paper, we show that the quantum solidification of ${ }^{4} \mathrm{He}$ can be considered variationally, with a single explicit wave-function which selects the phase through optimization of the thermodynamic potential. Quite surprisingly, we find that the phase transition is predicted properly, given the relative simplicity of the wave function. While the variational treatment is used for quantum phase transitions at the mean-field level [14], it is relatively uncommon that a (discontinuous) transition can be described with a microscopic wave function. The finding is also interesting in light of growing interest to the first-order quantum phase transitions $[15,16]$.

At zero temperature, the phases of ${ }^{4} \mathrm{He}$ can be studied in an essentially exact form with a family of projector methods, including Green's-function Monte Carlo [17,18], diffusion Monte Carlo [19], and path-integral ground-state Monte Carlo [20]. These methods properly describe the phase transition in helium, and can provide insight on the nature of its ground state $[21,22]$.

Variational calculations with shadow-type wave functions (SWFs) [23] provide accurate results both for the liquid and

\footnotetext{
*yaroslav.lutsyshyn@uni-rostock.de; www.physik.uni-rostock.de/qtmps

†http://bqmc.upc.edu
}

solid phases of ${ }^{4} \mathrm{He}[23,24]$, and describe the transition [25-27] and coexistence [28] between the two phases. The SWFs can be seen as representing a single step of a projection calculation [23]. The projection is carried out by performing the numerical integration of the shadow degrees of freedom. In this sense, the SWF is not fully explicit, as one cannot write down the result of such an integration. We consider SWF calculations as a class of their own, in between the exact projection methods and the simple and fully explicit wave function used here.

Highly effective wave functions have been developed over the years for liquid and (nonsymmetric) solid helium. Accurate multiparameter two- and three-body terms [29-31] result in energies that are nearly exact [31]. However, efficient one-body (lattice) terms that are also exchange-symmetric have not been reported.

The wave function that we consider here was proposed specifically for solid ${ }^{4} \mathrm{He}$ [32]. It has been since then used extensively for importance sampling in projector Monte Carlo methods [33-35]. This wave function is a product of the Jastrow term, which accounts for the pair correlations, and a cleverly symmetrized Nosanow-like term. The wave function has the form

$$
\psi_{\mathrm{snj}}=\left(\prod_{i<j}^{N_{\mathrm{p}}} f\left(\left|\boldsymbol{r}_{i}-\boldsymbol{r}_{j}\right|\right)\right)\left(\prod_{k}^{N_{\mathrm{s}}} \sum_{i}^{N_{\mathrm{p}}} g\left(\left|\boldsymbol{r}_{i}-\boldsymbol{l}_{k}\right|\right)\right),
$$

where $N_{\mathrm{p}}$ is the number of atoms, and $N_{s}$ is the number of lattice sites, located at $\boldsymbol{l}_{k}$. The position of the $i$ th particle is labeled $\boldsymbol{r}_{i}$. Suitable for our interest in the thermodynamic limit, $\psi_{\mathrm{snj}}$ has the translational invariance broken by the lattice site locations $\boldsymbol{l}_{k}$. Notice that the second, product-sum term in Eq. (1), is not a permanent, and the computational cost of $\psi_{\text {snj }}$ scales only as the square of the number of particles. Pair-correlation factors $f\left(r_{i j}\right)$ can be taken with the pseudopotential either in the McMillan form [36,37],

$$
f(r)=\exp \left[-\frac{1}{2}\left(\frac{b}{r}\right)^{5}\right]
$$

or in a more involved form with midrange correlations, as detailed below. Atoms are localized to the lattice sites with factors $g(r)$. We use the Gaussian form

$$
g(r)=\exp \left[-\frac{1}{2} \gamma r^{2}\right]
$$



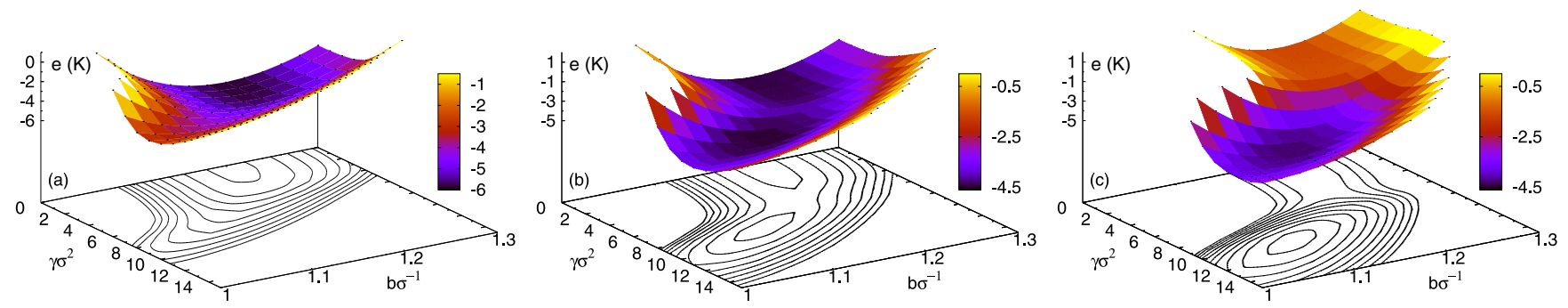

FIG. 1. (Color online) Energy per particle obtained with the two-parameter symmetric wave function $\psi_{\text {snj }}^{\{b, \gamma\}}$ given by Eqs. (1)-(3), as a function of variational parameters $b$ and $\gamma$, for three different densities. Parameters are shown in terms of $\sigma=2.556 \AA$. Simulation used $N_{\mathrm{p}}=N_{\mathrm{s}}=900$ particles. (a) Density $\rho=22.2 \mathrm{~nm}^{-3}$ displays a single minimum, at $\gamma=0$, which corresponds to a liquid phase; (b) intermediate density $\rho=25.8 \mathrm{~nm}^{-3}$ is in the liquid-solid coexistence region, and has two separate local minima; (c) density $\rho=29.3 \mathrm{~nm}{ }^{-3}$ displays only one minimum, corresponding to the solid phase. Contours are separated by $0.4 \mathrm{~K}$.

with parameter $\gamma$ describing the strength of the site localization.

To better understand the structure of $\psi_{\text {snj }}$, we can write the wave function in the form

$$
\psi_{\mathrm{snj}}=\prod_{i<j}^{N_{\mathrm{p}}} f\left(r_{i j}\right) \prod_{k}^{N_{\mathrm{s}}} S_{k},
$$

with the site sums $S_{k}\left(\boldsymbol{r}_{1}, \ldots, \boldsymbol{r}_{N_{\mathrm{p}}}\right)$ given by

$$
S_{k}=\sum_{i}^{N_{\mathrm{p}}} g\left(\left|\boldsymbol{r}_{i}-\boldsymbol{l}_{k}\right|\right) \text {. }
$$

While each sum $S_{k}$ depends on the coordinates of all particles, it does not contain interparticle distances. One can view them as a generalized form of one-body correlation factors, in the formal sense that $\nabla_{i \neq j} \cdot \nabla_{j} S_{k}=0$. In this view, Eq. (4) consists of the one- and two-body terms of the general Feenberg form for the trial wave function [38,39]. Equation (4) also emphasizes the flexibility of $\psi_{\text {snj }}$. The number of sites does not need to be equal to the number of particles. One may confine atoms to given regions of the lattice by including these atoms only in some of the sums $S_{k}$. Limiting each sum to only one atom recovers the original Nosanow-Jastrow wave function [40], with $N_{\mathrm{s}}=N_{\mathrm{p}}$,

$$
\psi_{\mathrm{nj}}=\left(\prod_{i<j}^{N_{\mathrm{p}}} f\left(\left|\boldsymbol{r}_{i}-\boldsymbol{r}_{j}\right|\right)\right)\left(\prod_{k}^{N_{\mathrm{s}}} g\left(\left|\boldsymbol{r}_{k}-\boldsymbol{l}_{k}\right|\right)\right) .
$$

The Nosanow-Jastrow wave function $\psi_{\text {nj }}$ yields good variational energy and has long been used to describe solid ${ }^{4} \mathrm{He}$. Unfortunately, $\psi_{\mathrm{nj}}$ is not exchange symmetric [41]. The onebody term imposes a heavy penalty for removing an atom away from its "parent" site. A straightforward symmetrization of $\psi_{\mathrm{nj}}$ yields poor results [42], or otherwise results in computationally prohibitive wave functions.

The lattice structure $\boldsymbol{l}_{k}$, which enters through the sitelocalization terms $g(\cdot)$, can in principle be seen as a parameter to the wave function. In this case one may optimize between different lattice symmetries, or optimize individual site positions. On the other hand, the lattice can be seen as an input to the problem. Here we follow the latter path, since we aim to study the experimentally known zero-temperature solid phase of ${ }^{4} \mathrm{He}$. Thus $\boldsymbol{l}_{k}$ are located on a geometrically ideal hcp lattice.
We begin with the variational energy optimization of the two-parameter trial wave function $\psi_{\mathrm{snj}}^{\{b, \gamma\}}$ given by Eqs. (1)-(3). The energy is given by

$$
E(b, \gamma)=\left\langle\psi_{\mathrm{snj}}^{\{b, \gamma\}}|\hat{H}| \psi_{\mathrm{snj}}^{\{b, \gamma\}}\right\rangle /\left\langle\psi_{\mathrm{snj}}^{\{b, \gamma\}} \mid \psi_{\mathrm{snj}}^{\{b, \gamma\}}\right\rangle,
$$

with many-body Hamiltonian

$$
\hat{H}=-\frac{\hbar^{2}}{2 m_{\mathrm{He} 4}} \sum_{i=1}^{N_{\mathrm{p}}} \nabla_{i}^{2}+\sum_{i \neq j} V\left(r_{i j}\right),
$$

using the pairwise interaction potential proposed by Aziz et al. [43]. The multidimensional integral implied by Eq. (6) was evaluated with a Metropolis Monte Carlo scheme [44,45]. We performed a direct minimization on a grid of $b$ and $\gamma$ values, for a range of densities. Three characteristic examples of the energy surface are shown in Fig. 1. At low densities, there is a single minimum with $\gamma=0$. The wave function with $\gamma=0$ reduces to the translationally invariant Jastrow product and corresponds to a liquid phase. At intermediate densities, an additional local minimum appears at nonzero values of $\gamma$, corresponding to a state with broken translational symmetry. This minimum corresponds to a crystalline phase, as was verified from the scaling with $N_{\mathrm{p}}$ of the static structure function. With further increase in density, this second $\gamma \neq 0$ minimum lowers in energy and eventually "overtakes" the liquid $\gamma=0$ minimum. Thus the solid phase becomes preferred variationally, and the optimized system loses translational symmetry. With the densities increased further still, the liquid minimum disappears. While this picture is quite similar to the classical first-order phase transition, where energy is considered as a function of thermodynamic parameters of the system, here we are plotting the energy surface as a function of variational parameters of the wave function. The optimal values of parameters $b$ and $\gamma$, shown in Fig. 2 as a function of density, display a clear transition between the solid and liquid states. As our simulated system is finite, the sharpness of this transition is in fact a remarkable occurrence [46-48]. Despite effort, we were not able to detect any smooth rollover between the phases. Technically, the two minima in the energy surface, as shown in Fig. 1(b), are always distinct. We attribute this effect to the fact that the thermodynamic limit is accessible to the finite system through the provided lattice $\boldsymbol{l}_{k}$.

Full thermodynamic analysis requires minimization of the Gibbs free energy, which at $T=0$ equals enthalpy, $G=E+$ 


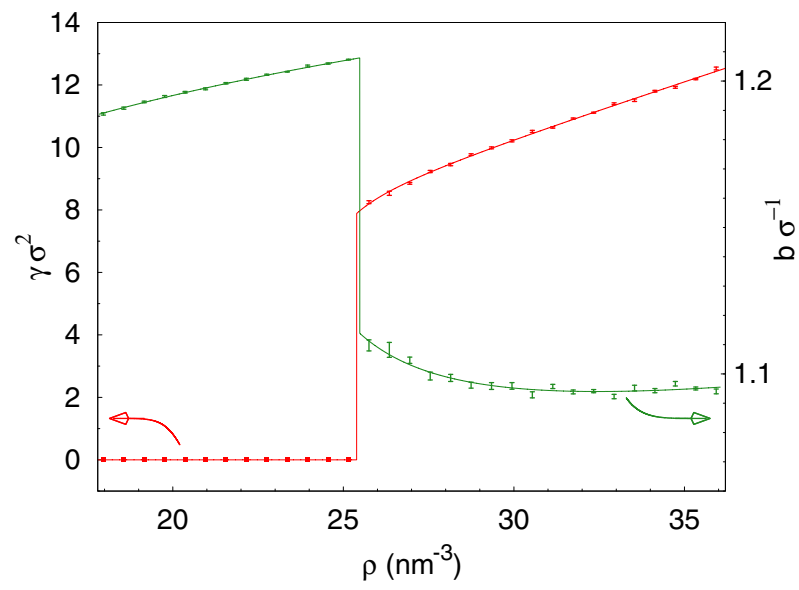

FIG. 2. (Color online) Optimized parameters of the twoparameter symmetric wave function given by Eqs. (1)-(3), as a function of density. Parameters are shown in units of $\sigma=2.556 \AA$. Left vertical axis corresponds to the value of the site-localization parameter $\gamma$, while the right axis shows parameter $b$.

$P V$. Enthalpy has to be extracted from the equations of state $E^{\{b, \gamma\}}(\rho)$ computed for each possible set of parameters $\{b, \gamma\}$. The pressure can be computed via

$$
P^{\{b, \gamma\}}(\rho)=\rho^{2} \frac{\partial E^{\{b, \gamma\}} / N}{\partial \rho} .
$$

We solve the above equation for $\rho^{\{b, \gamma\}}(P)$ and find the Gibbs energy for each set of parameters,

$$
G^{\{b, \gamma\}}(P)=E\left[\rho^{\{b, \gamma\}}(P)\right]+P N / \rho^{\{b, \gamma\}}(P) .
$$

Next, we minimize $G^{\{b, \gamma\}}(P)$ with respect to variational parameters,

$$
G(P)=\min _{\{b, \gamma\}} G^{\{b, \gamma\}}(P) .
$$

It is possible to show that only the parameters which minimize energy at some density will also minimize free energy at any pressure. The parameters which minimize the free energy at a given pressure also provide the density and energy at that pressure. While this method is relatively straightforward, such analysis has not been reported in the past, presumably because of the large underlying computational costs.

We carried out the minimization procedure outlined above for a range of densities. The Gibbs free energy, shown in Fig. 3 as a function of pressure, exhibits a kink characteristic of a first-order phase transition. Corresponding to the weakness of this transition, the kink is subtle yet well defined. We performed a linear fit to the free energy of the solid phase near the transition, and subtracted this fit from the free energies. The result, which emphasizes the transition, is plotted in the inset to Fig. 3. Somewhat unexpectedly, the transition occurs at a pressure of $20 \mathrm{~atm}$, close to the correct value of $25 \mathrm{~atm}$. This is especially surprising given the simplicity of our two-parameter wave function. The optimization of the Gibbs energy at a given pressure also selects the density of the state. This is equivalent to inverting Eq. (7) for the density $\rho$ under the minimization condition of Eq. (8). At the transition pressure, optimized density jumps from the lower density of freezing $\rho_{f}$ to the higher density of melting $\rho_{m}$ [49]. At zero temperature,

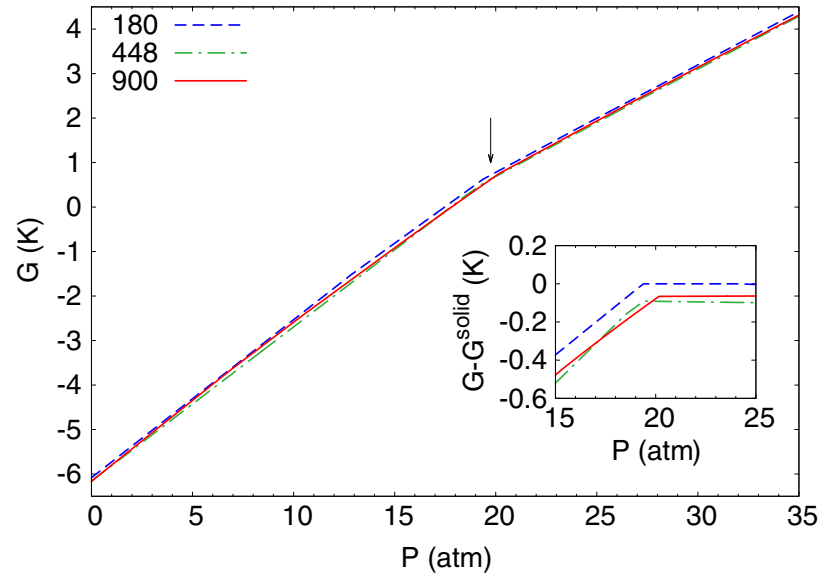

FIG. 3. (Color online) Gibbs free energy, per particle, of the optimized state of the two-parameter symmetric wave function given by Eqs. (1)-(3). Arrow indicates the location of the phase transition. The inset shows the free energies with subtracted linear fit to the solid phase of the 180-particle system.

the density discontinuity also provides information about the latent heat $\Delta E$, since $\Delta E=P\left(1 / \rho_{f}-1 / \rho_{m}\right)$. Optimized density $\rho(P)$ is plotted in Fig 4, along with the experimental values [50]. Results are shown for several particle numbers, from $N_{\mathrm{p}}=180$ to $N_{\mathrm{p}}=900$. As can be seen, the size effects are moderate and the extrapolated transition pressure for the two-parameter wave function is close to $20 \mathrm{~atm}$.

The two-body factors of Eq. (2) account for the short-range behavior of the interaction potential. More accurate two-body

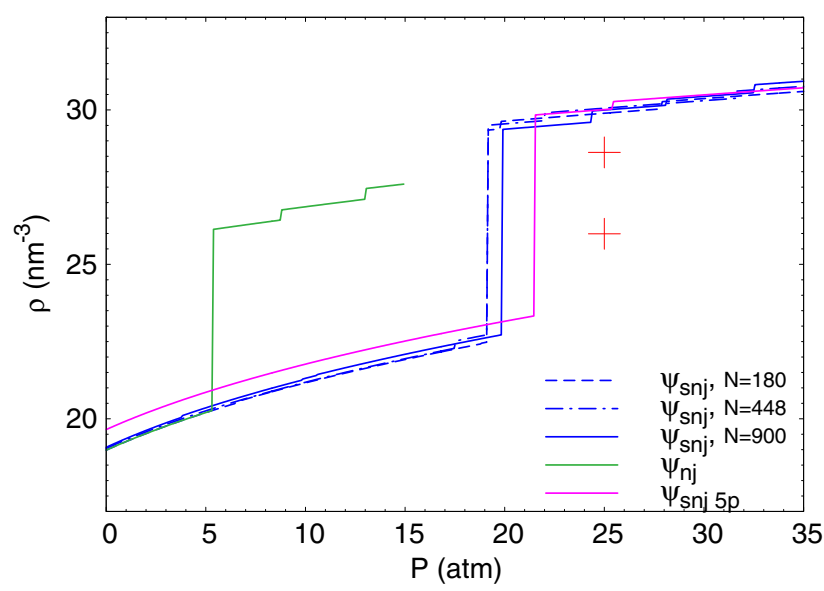

FIG. 4. (Color online) Optimized density as a function of pressure. Blue lines show density for the two-parameter symmetric wave function $\psi_{\text {snj }}$ for varying number of particles, $N=180,448$, and 900. The size effects in the transition location are below the statistical error, which is about $1 \mathrm{~atm}$. Purple line shows density for five-parameter symmetric wave function with improved pair-correlation factor (9), with transition at 22 bars. Green line shows density for the traditional, nonsymmetric Nosanow-Jastrow wave function of Eq. (5), with transition at 5 bars. Red cross marks show experimental melting and freezing densities [50]. The piecewise appearance of data comes from different equations of state $\rho(P)$ selected by the varying pressure-optimized variational parameters; size of the steps is thus indicative of the statistical error. 
factors can be obtained by including mid-range correlations, as in the form proposed in Ref. [51],

$$
f(r)=\exp \left[-\frac{1}{2}\left(\frac{b}{r}\right)^{5}+\frac{1}{2} s \exp \left(\frac{r-\lambda}{w}\right)^{2}\right] .
$$

The resulting five-parameter symmetric wave function given by Eqs. (1), (3), and (9) was optimized and analyzed as described above. For nonzero optimal $\gamma$, the midrange correlation factor optimizes away, i.e., $s=0$. That is, the solid phase does not benefit from such correlations. The results for the optimal density at each pressure are shown in Fig. 4. As can be seen, the transition location has improved, and the zero-pressure liquid density has increased, which is closer to the experiment. We also carried the optimization with the two-body factors that included the low-energy phonon contribution along Ref. [52]. Such terms had little influence on the transition location. We conclude that the main source of discrepancy in the pressure of the phase transition stems from deficiencies in the two-body factors and the absence of three-body correlation factors in the liquid phase.

The optimization of the thermodynamic potential was also carried out for the two-parameter (unsymmetrized) NosanowJastrow wave function $\psi_{\mathrm{nj}}$ given by Eqs. (2), (3), and (5). The optimization results are included in Fig. 4. Solidification for $\psi_{\text {nj }}$ occurs already at 5 bars, a dramatic fivefold departure from the correct location of the transition. This coincides with early findings by Hansen and Levesque, who determined the transition for the unsymmetrized wave function to occur at pressures of about 8 atm [53]. In fact, the freezing density $\rho_{f}$ for $\psi_{n j}$ lies below the correct experimental equilibrium density of helium liquid at vapor pressure. (The equilibrium density is underestimated by all wave functions by as much as $15 \%$.) Thus the nonsymmetric Nosanow-Jastrow wave function misses the transition by a wide margin. It should be noted that $\psi_{\mathrm{nj}}$ in fact provides lower energies for the solid than the symmetric $\psi_{\text {snj }}$, by up to $1 \mathrm{~K}$. Strict site localization makes $\psi_{\mathrm{nj}}$ insensitive to the deficiencies in the two-body factors. The symmetrical $\psi_{\mathrm{snj}}$ allows for virtual interstitials and is more sensitive to the form of two-body factor $f(r)$. As the balance between phases amounts to the difference between free energies, to some extent a cancellation occurs, improving the location of the transition for $\psi_{\mathrm{snj}}$.

To summarize, we studied at the variational level the quantum phase transition between superfluid and hcp solid ${ }^{4} \mathrm{He}$. The transition properties were determined by optimizing the Gibbs free energy. We used a wave function which describes a quantum solid with broken translational symmetry but that is, at the same time, exchange symmetric. Below the melting pressure, the optimized wave function reduces to a translationally symmetric Jastrow function describing a liquid. Given the simplicity of the wave function, it is remarkable that the transition is found at a pressure that is only three to five atm away from the correct experimental value. We attribute the discrepancy to the quality of the pair-correlation terms and the lack of three-body correlations in the liquid phase. These findings strongly support the form given by Eq. (1) as a suitable symmetric wave function for describing both a first-order quantum phase transition and a quantum Bose solid.

Authors thank the Barcelona Supercomputing Center (The Spanish National Supercomputing Center-Centro Nacional de Supercomputación) for the provided computational facilities. We acknowledge partial financial support from DGI (Spain) Grant No. FIS2011-25275 and Generalitat de Catalunya Grant No. 2009SGR-1003. G.E.A. acknowledges support from the Spanish MEC through the Ramon y Cajal fellowship program.
[1] E. Kim and M. H. W. Chan, Nature (London) 427, 225 (2004).

[2] E. Kim and M. H. W. Chan, Science 305, 1941 (2004).

[3] J. Day and J. Beamish, Nature (London) 450, 853 (2007).

[4] X. Rojas, A. Haziot, V. Bapst, S. Balibar, and H. J. Maris, Phys. Rev. Lett. 105, 145302 (2010).

[5] J. D. Reppy, Phys. Rev. Lett. 104, 255301 (2010).

[6] M. W. Ray and R. B. Hallock, Phys. Rev. B 84, 144512 (2011).

[7] Y. Vekhov and R. B. Hallock, Phys. Rev. B 90, 134511 (2014).

[8] M. H. W. Chan, R. B. Hallock, and L. Reatto, J. Low Temp. Phys. 172, 317 (2013).

[9] M. Boninsegni, L. Pollet, N. Prokof'ev, and B. Svistunov, Phys. Rev. Lett. 109, 025302 (2012).

[10] I. Pomeranchuk, JETP 20, 919 (1950).

[11] R. C. Richardson, Rev. Mod. Phys. 69, 683 (1997).

[12] B. K. Clark, M. Casula, and D. M. Ceperley, Phys. Rev. Lett. 103, 055701 (2009).

[13] T. Minoguchi, M. Nava, F. Tramonto, and D. Galli, J. Low Temp. Phys. 171, 259 (2013).

[14] S. Sachdev, Quantum Phase Transitions (Cambridge University Press, Cambridge, England, 2001).

[15] M. Macek and A. Leviatan, Ann. Phys. 351, 302 (2014).

[16] C. Pfleiderer, J. Phys.: Condens. Matter 17, S987 (2005).
[17] P. A. Whitlock, D. M. Ceperley, G. V. Chester, and M. H. Kalos, Phys. Rev. B 19, 5598 (1979).

[18] M. H. Kalos, M. A. Lee, P. A. Whitlock, and G. V. Chester, Phys. Rev. B 24, 115 (1981).

[19] L. Vranješ, J. Boronat, J. Casulleras, and C. Cazorla, Phys. Rev. Lett. 95, 145302 (2005)

[20] A. Sarsa, K. E. Schmidt, and W. R. Magro, J. Chem. Phys. 113, 1366 (2000).

[21] G. L. Masserini and L. Reatto, Phys. Rev. B 35, 6756 (1987).

[22] C. Mora and X. Waintal, Phys. Rev. Lett. 99, 030403 (2007).

[23] S. Vitiello, K. Runge, and M. H. Kalos, Phys. Rev. Lett. 60, 1970 (1988).

[24] S. Moroni, D. E. Galli, S. Fantoni, and L. Reatto, Phys. Rev. B 58, 909 (1998).

[25] F. Pederiva, A. Ferrante, S. Fantoni, and L. Reatto, Phys. Rev. B 52, 7564 (1995).

[26] B. Krishnamachari and G. V. Chester, Phys. Rev. B 61, 9677 (2000).

[27] T. MacFarland, G. V. Chester, M. H. Kalos, L. Reatto, and S. A. Vitiello, Phys. B (Amsterdam, Neth.) 194-196, 525 (1994).

[28] F. Pederiva, A. Ferrante, S. Fantoni, and L. Reatto, Phys. Rev. Lett. 72, 2589 (1994). 
[29] V. R. Pandharipande and H. A. Bethe, Phys. Rev. C 7, 1312 (1973).

[30] S. A. Vitiello and K. E. Schmidt, Phys. Rev. B 46, 5442 (1992).

[31] S. A. Vitiello and K. E. Schmidt, Phys. Rev. B 60, 12342 (1999).

[32] C. Cazorla, G. E. Astrakharchik, J. Casulleras, and J. Boronat, New J. Phys. 11, 013047 (2009).

[33] C. Cazorla and J. Boronat, Phys. Rev. B 88, 224501 (2013).

[34] C. Cazorla, Y. Lutsyshyn, and J. Boronat, Phys. Rev. B 87, 214522 (2013).

[35] Y. Lutsyshyn, C. Cazorla, G. E. Astrakharchik, and J. Boronat, Phys. Rev. B 82, 180506 (2010).

[36] W. L. McMillan, Phys. Rev. 138, A442 (1965).

[37] D. Schiff and L. Verlet, Phys. Rev. 160, 208 (1967).

[38] E. Feenberg, Ann. Phys. 84, 128 (1974).

[39] C. C. Chang and C. E. Campbell, Phys. Rev. B 15, 4238 (1977).

[40] L. H. Nosanow, Phys. Rev. Lett. 13, 270 (1964).

[41] One can, in principle, argue that the overlap of any nonsymmetrical wave function and the true ground state of ${ }^{4} \mathrm{He}$ scales with the number of particles $N$ as $(N !)^{-1}$.

[42] D. Ceperley, G. V. Chester, and M. H. Kalos, Phys. Rev. B 17, 1070 (1978).

[43] R. A. Aziz, F. R. W. McCourt, and C. C. K. Wong, Mol. Phys. 61, 1487 (1987).
[44] Y. Lutsyshyn, Comp. Phys. Comm. 187, 162 (2015).

[45] We employed a CUDA parallelization for a graphical processing unit as described in Ref. [44], and used a cluster of Nvidia GPU to carry out the integrals of Eq. (6) to a sufficiently high accuracy. All calculations were carried out to at least $10 \mathrm{mK}$ error in energy per particle.

[46] M. Campostrini, J. Nespolo, A. Pelissetto, and E. Vicari, Phys. Rev. Lett. 113, 070402 (2014).

[47] M. Mueller, W. Janke, and D. A. Johnston, Phys. Rev. Lett. 112 200601 (2014).

[48] G.-D. Lin, C. Monroe, and L.-M. Duan, Phys. Rev. Lett. 106, 230402 (2011).

[49] The density of freezing $\rho_{f}$ is the lowest density at which the liquid can start to freeze, that is, to coexist with solid. The density of melting $\rho_{m}$ is the density at which the solid begins to melt, that is, to coexist with liquid. Somewhat unintuitively, $\rho_{f}<\rho_{m}$. The phase coexistence region lies in the densities $\rho_{f}<\rho<\rho_{m}$.

[50] E. R. Grilly, J. Low Temp. Phys. 11, 33 (1973).

[51] L. Reatto, Nucl. Phys. A 328, 253 (1979).

[52] W. P. Francis, G. V. Chester, and L. Reatto, Phys. Rev. A 1, 86 (1970).

[53] J.-P. Hansen and D. Levesque, Phys. Rev. 165, 293 (1968). 